\title{
Efficiency and fidelity of molecular simulations relevant to dislocation evolutions
}

\author{
Fei Shuang ${ }^{\mathrm{a}, \mathrm{b}}$, Pan Xiao ${ }^{\mathrm{a}, *}$, Fujiu $\mathrm{Ke}^{\mathrm{c}}$, Yilong Bai ${ }^{\mathrm{a}}$ \\ ${ }^{a}$ LNM, Institute of Mechanics, Chinese Academy of Sciences, Beijing 100190, China \\ ${ }^{\mathrm{b}}$ School of Engineering Sciences, University of Chinese Academy of Sciences, Beijing 100049, China \\ ' School of Physics and Nuclear Energy Engineering, Beihang University, Beijing 100191, China
}

\section{A R T I C L E I N F O}

\section{Article history:}

Received 25 May 2017

Received in revised form 30 July 2017

Accepted 31 July 2017

Available online 17 August 2017

\section{Keywords:}

Molecular dynamics

Energy minimization

Dislocation evolution

Computational efficiency

\begin{abstract}
A B S T R A C T
The comparative simulations of dislocation evolution in nanoindentation are carried out in terms of both molecular dynamics (MD) and energy minimization (EM) methods, to explore what really govern the computational efficiency and fidelity in molecular simulations relevant to dislocation evolutions. It is found that although all simulations can present similar relationship between indentation force and depth, there still might be some significant differences in the simulated dislocation patterns and computational efficiency. Firstly, the EM simulations show more complicated dislocations. Secondly, the necessary computational effort of EM increases nonlinearly with indentation depth, compared to the linear dependence in MD simulations, namely EM shows higher efficiency than MD in shallow indentation, but vice versa in deeper ones. More importantly, it is revealed that the time consumption of the minimization iteration is strongly dependent on the moving of dislocation loops and increases greatly when dislocation loops move long distances. Whereas MD simulations of complicated dislocations patterns may need less time cost but present immature dislocation evolutions, since the relaxation steps in MD simulations are fixed beforehand, regardless of the dislocation loops moving to equilibrium state or not.
\end{abstract}

(c) 2017 Elsevier B.V. All rights reserved.

\section{Introduction}

Dislocation evolution is key microscopic event for understanding plasticity, damage and failure of crystal materials and structures. It is a long pursuit to gain both high efficiency and high fidelity in large scale molecular simulations of dislocation evolutions. Molecular dynamics (MD) and energy minimization (EM) methods are two of the commonly used approaches [1-5]. MD, based on Newton's second law of motion for atoms, has been widely used to describe dynamic behavior of dislocations in nanoscale materials $[1,2,6]$. However, the time scale of MD is limited to several nanoseconds with loading strain rate usually higher than $10^{8} \mathrm{~s}^{-1}$ [7]. EM, on the other hand, based on minimization of a given system's energy, is usually used to investigate dislocation evolutions at quasi-static conditions $[3,4]$.

Molecular statics (MS) is a typical EM method with potential energy as the objective of minimization. It has been applied to structural optimization of biomacromolecules [8] and quasi-static simulation of solid atomistic systems [3,4]. Kang and Huang have successfully used MS to investigate dislocation evolution in copper

\footnotetext{
* Corresponding author.

E-mail address: xiaopan@Inm.imech.ac.cn (P. Xiao).
}

nanowires under tensile, rotating and shear deformations [9]. However, temperature of the system simulated using MS is limited to $0 \mathrm{~K}$. EM can be extended to handle systems at finite temperature if free energy is used as objective of minimization. For example, the molecular statistical thermodynamics (MST) method proposed by Hu et al. uses the statistical thermodynamics formulation of Helmholtz free energy of molecular system [10]. Applications of MST indicate that it is an effective approach for simulating dislocation evolution in nanostructures under quasi-static deformation at finite temperature $[11,12]$.

In order to simulate dislocation evolution in large scale molecular systems, multi-scale methods based on EM have also been proposed in the last few decades. In multi-scale simulations, atomistic representation is used in regions under inhomogeneous deformation dominated by dislocation evolutions to capture atomistic details, while quasi-continuum representation is used in regions under homogeneous deformation without dislocation to reduce computational costs. One of the typical multi-scale methods is the quasi-continuum method $(\mathrm{QC})$ which solves the positions of representative atoms by minimize the coarse-grained potential energy of the system at temperature $0 \mathrm{~K}$ [13]. Based on the framework of EM, some other multi-scale methods such as coupling of length scales method [14], bridging domain method 
[15], bridging scale method [16], composite grid atomistic continuum method [17] and multi-resolution molecular statics [18] have also been proposed and applied for studies of dislocation evolution in large scale systems [19]. Based on MST, Wang et al. proposed the hybrid molecule/cluster statistical method [10] which is a multi-scale approach for simulating dislocation evolution in nanostructures at finite temperature. Chen and Ming [20,21] proposed a constrained Cauchy-Born elasticity accelerated multigrid method and reduced considerable computational costs in nanoindentation with simple dislocations. Although so many multi-scale methods have been proposed in the past years, few of them have been widely used. One of the main reasons is that efficiency improvement of some EM-based multi-scale methods against MD in practice is not remarkable. Therefore, what really governs the computational efficiency and how to improve it should be further investigated.

Although both MD and EM-based simulations have been performed to investigate dislocation evolution in molecular systems at different conditions, the computational efficiency and fidelity of the two methods is rarely studied systematically. A deep investigation of the problem will provide useful information for simulations relevant to dislocation evolutions in large scale molecular systems regarding (i) making choice between MD and EM-based methods for a specific problem, (ii) modifying related settings in MD and EM-based methods in order to obtain reasonable results, (iii) understanding bottle-neck of computational efficiency in EM-based multi-scale methods and, (iv) developing new algorithms to accelerate the simulation relevant to dislocation evolutions. In this work, the comparative simulations of dislocation evolution in nanoindentation were carried out in terms of both MD and MS, to explore what really govern the computational efficiency and fidelity in molecular simulations of dislocation evolutions.

\section{Computational framework}

The MD and MS simulations are performed on a nanoindentation system consisting of a substrate material indented by a conical diamond tip, as illustrated in Fig. 1. Nanoindentation is a flexible characterization technique in mechanical analysis of nanostructures. Simulation of nanoindentation has been commonly used as a benchmark to examine new multi-scale methods because $[22,23]$, (i) it provides dislocation evolution details for understanding mechanical behavior of materials at nanoscale; (ii) complex dislocation nucleation and evolution can be trigged by indentation

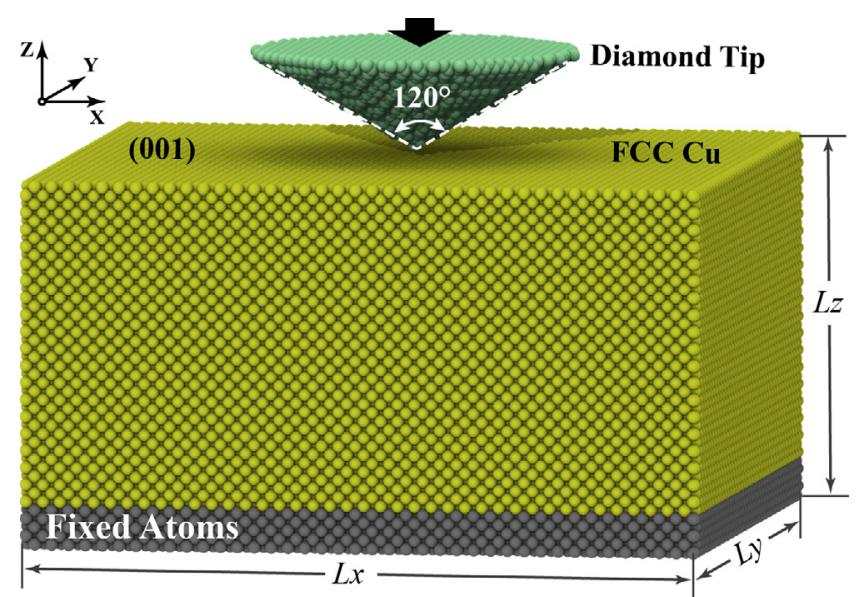

Fig. 1. Configuration of the nanoindentation system consisting of $\mathrm{Cu}$ substrate and diamond tip used in MD and MS simulations. tip easily and new methods are examined whether they could capture these changes and; (iii) relation of indentation force and depth obtained from experiments and large scale simulations can be compared directly.

The substrate in Fig. 1 consists of facial cubic crystal (FCC) $\mathrm{Cu}$ with lattice constant of $3.615 \AA$. The dimensions of the substrate $(L x \times L y \times L z)$ is $43.4 \times 43.4 \times 21.7 \mathrm{~nm}$ with $3,542,000$ atoms. The free surface at the top is $\left(\begin{array}{lll}0 & 0 & 1\end{array}\right)$ crystal plane and the other two side surface are $\left(\begin{array}{lll}1 & 0 & 0\end{array}\right)$ and $\left(\begin{array}{lll}0 & 1 & 0\end{array}\right)$. Periodic boundary conditions are imposed against the side surfaces to approximate a nanofilm with infinite width. Atomistic layers with depth of $1.5 \mathrm{~nm}$ at the bottom are fixed to provide a support in $z$ direction. The substrate is indented by a conical-shaped indenter tip consists of 149,683 C atoms of diamond lattice. In both MD and MS simulations, the tip moves against the top surface of the substrate with $0.01 \mathrm{~nm}$ for each loading step. The maximum indentation depth is $2.53 \mathrm{~nm}$.

The embedded atom model (EAM) potential developed by Mishin et al. [24] is used for Cu atoms. The potential has been widely applied to simulate mechanical responses of FCC metals and reasonable results have been obtained in comparison with experimental measurements [25]. Interactions between $C$ atoms in the tip and $\mathrm{Cu}$ atoms in the substrate are modeled by a Morse potential [12]

$\boldsymbol{\Phi}=D_{0}\left[e^{-2 \alpha\left(r-r_{0}\right)}-2 e^{-\alpha\left(r-r_{0}\right)}\right]$,

with parameters $D_{0}=0.087 \mathrm{eV}, \alpha=5.14 \AA^{-1}, r_{0}=2.05 \AA$. The indenter tip is assumed to be rigid during simulations, so interactions between $\mathrm{C}$ atoms is ignored.

In MS simulations, total potential energy of the system at each loading step is minimized using conjugate gradient (CG) algorithm. In CG algorithm [26], atomistic position vector $\left(\mathbf{x}_{k}\right)$ is updated according to the current conjugate direction $\left(\mathbf{d}_{k}\right)$ and step size $\left(\alpha_{k}\right)$ :

$\mathbf{x}_{k+1}=\mathbf{x}_{k}+\alpha_{k} \mathbf{d}_{k}$,

where $k$ indicates the iteration step of conjugate directions. $\mathbf{d}_{k}$ is calculated from atomistic force vector $\left(\mathbf{f}_{k}\right)$ and searching direction. At each loading step, there will be different number of iteration steps $\left(n_{\text {iter }}\right)$ which is related to characteristics of energy profiles. At iteration step $k, \alpha_{k}$ is determined through line-searching methods in which the system's energy and atomistic force vector needs to be evaluated for several times $\left(n_{\text {line }}^{k}\right)$. Therefore, the total times of energy and force evaluation $\left(n_{\text {eval }}\right)$ at a loading step is

$n_{\text {eval }}=\sum_{k=1}^{n_{\text {iter }}} n_{\text {line }}^{k}$.

The system is assumed to reach an equilibrium state when the resolution criterion $(\varepsilon)$ of minimization is satisfied:

$\max \left(e_{k+1}^{i}-e_{k}^{i}\right)<\varepsilon, \quad i=1,2, \ldots, N$

where $N$ is the total of atoms, $e_{k+1}^{i}$ and $e_{k}^{i}$ are the per-atom potential energies at the $k+1$ and $k$ iteration step respectively. In this work, $\varepsilon$ with values of $1 \times 10^{-3}, 1 \times 10^{-4}$ and $1 \times 10^{-5} \mathrm{eV}$ is considered in order to investigate its effect on simulation results.

In experiments, nanoindentation is usually performed with loading velocity slower than $1 \mu \mathrm{m} / \mathrm{s}$ which can be assumed quasi-static. The quasi-static process can be modeled effectively using MS; however it is hard to perform a quasi-static simulation with MD whose intrinsic time scale is about $1 \mathrm{fs}$. In practice, at each loading step, the system is relaxed with pre-specified number of MD time-steps $\left(n_{r}\right)$ to equivalent a quasi-static process. At each time-step, atomistic position vector $\mathbf{x}_{k}$ is updated according to the current velocity and force vectors:

$\mathbf{x}_{k+1}=\mathbf{x}_{k}+\boldsymbol{v}_{k} \Delta t+\frac{\Delta t^{2}}{2 m} \mathbf{f}_{k}$ 
where $\Delta t$ is the timestep which is set to be $2 \mathrm{fs}$ in our simulations. So the real relaxation time $\left(t_{r}\right)$ for each loading step is $n_{r} \Delta t$. Simulations with $t_{r}$ ranging from 0.2 to $10 \mathrm{ps}$ are performed to examine the influence of $t_{r}$ on the efficiency and fidelity of MD simulations relevant to dislocation evolutions. In order to compare results with that of MS, NVT ensemble is adopted in MD and the temperature is set close to $0 \mathrm{~K}$. All the MD simulations are performed using the open-source LAMMPS code [27].

Although all MS and MD simulations are performed on the same cluster with the same number of processors, it is inappropriate to compare computational efficiency based on CPU time directly. MS and MD are different in algorithms. CPU time spent on iteration, update of atomic attributes and data communication among processors is quite different. However, the most time-consuming computation in both MS and MD simulations is the same: the calculation of system's energy and atomistic force vector which needs to traverse all atom pairs within a given cutoff distance. Therefore, total times of force evaluation $\left(n_{\text {eval }}\right)$ is used to compare the computational costs in MS and MD simulations. At each loading step, $n_{\text {eval }}=n_{r}$ in MD, while $n_{\text {eval }}$ in MS is determined by Eq. (3).

\section{Results and discussion}

\subsection{Results of MD and MS simulations of dislocation evolution}

Relationship between indentation force $(F)$ and depth $(h)$ can be obtained directly from nanoindentation experiments. Based on the relationship, mechanical properties, such as hardness, Young's modulus of the substrate material can be extracted. Therefore, the $F-h$ relationship obtained from simulations is usually examined to see whether simulation result is reasonable. $F$ - $h$ curves obtained in MS simulation with $\varepsilon=10^{-5} \mathrm{eV}$ and MD simulations with $t_{r}=3 \mathrm{ps}$ and $10 \mathrm{ps}$ are plotted in Fig. 2(a) respectively. Generally, all the $F$ - $h$ curves are similar to the variation trends obtained from nanoindentation experiments. Before point A $(h=0.31 \mathrm{~nm})$ in Fig. 2 (a), all the three curves are coincident with each other. It is corresponding to elastic indentation stage. After that, $F$ increases with mini drops which are caused by dislocation events in the substrate material [28]. Although all the three $F$ - $h$ curves are very close to each other, the computational cost indicated by the accumulated $n_{\text {eval }}$ (denoted as $N_{\text {eval }}$ in Fig. 2(b)) over all loading steps from the three simulations is significantly different. Specifically, $N_{\text {eval }}$ is linearly dependent on $h$ in MD simulations; while in MS simulation, $N_{\text {eval }}$ increases nonlinearly with $h$. Computational cost of the MD simulation with $t_{r}=10 \mathrm{ps}$ is always larger than that of MS. For the MD simulation with $t_{r}=3 \mathrm{ps}$, when $h<1.58 \mathrm{~nm}$ (point $C$ in Fig. 2(b)), the computational cost is larger than MS, but vice versa in deeper $h$. Dislocation distributions at the maximum indentation depth $(h=2.53 \mathrm{~nm})$ obtained from MD $\left(t_{r}=3 \mathrm{ps}\right)$ and MS $\left(\varepsilon=10^{-5} \mathrm{eV}\right)$ are also different, as shown in Fig. 2(c). All the dislocation is identified using the centrosymmetry parameters (CSP) [29]. Three types of dislocation can be observed in the substrate: complex dislocation clusters near the indenter tip, prismatic dislocation loops (PDLs) and V-type dislocations at the upper surfaces. The volume, quantity and shape of the three types of dislocation obtained from MS are greatly different from that of MD. Particularly, there are more PDLs in the substrate in MS simulation.

\subsection{Effect of dislocation evolution on computational cost of MS simulations}

In order to understand the nonlinear dependency of computational cost on $h$ in MS simulations, $n_{\text {eval }}$ of MD and MS simulations at each loading step is plotted in Fig. 3(a). Apparently, $n_{\text {eval }}$ of MD for each loading step is constant since the relaxation time is
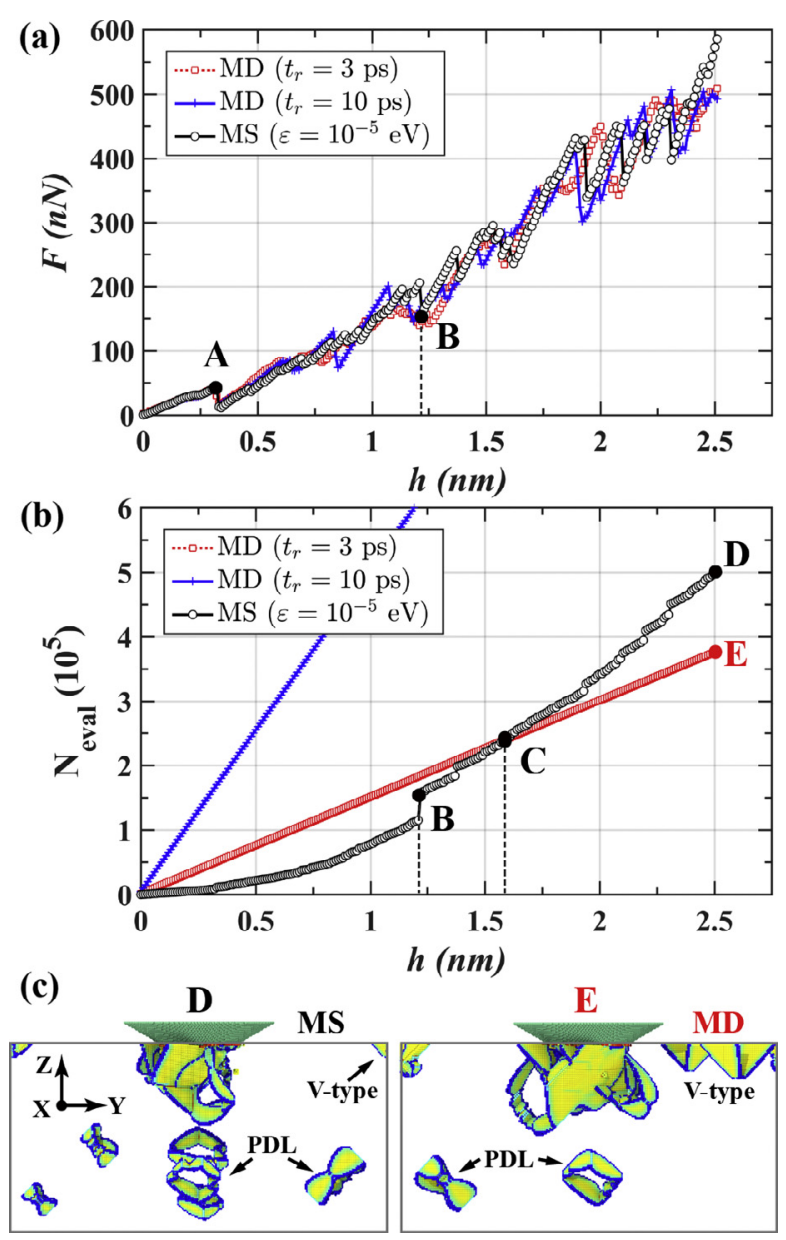

Fig. 2. (a) Indentation force and (b) accumulated times of force evaluation versus indentation depth obtained in MD and MS simulations. (c) Dislocation distribution obtained in MD and MS simulations at the last loading step.

pre-specified. The atomistic coordinate updating formula (5) of MD has nothing to do with dislocations. In another word, MD simulations do not "feel" and "care" about microstructure evolution. However, $n_{\text {eval }}$ of MS varies significantly with $h$ and there are a lot of $n_{\text {eval }}$ peaks. Particularly, at point B in Fig. $3(\mathrm{a})(h=1.22 \mathrm{~nm})$, it takes 41,892 times of force evaluation for MS to reach an equilibrium state which is much larger than that of the other steps. Fig. 3 (b) and (c) show dislocation patterns before and after minimization at loading step B. Before and after minimization, three significant modes of dislocation evolution can be detected: (I) a V-type dislocation moves from P1 to P2 on the top surface along [ $\left.\begin{array}{lll}1 & 1 & 0\end{array}\right]$ direction with distance of about $19.95 \mathrm{~nm}$; (II) a PDL is emitted from the dislocation clusters under the indenter tip and propagates from Q1 to Q3 along [ $01 \overline{1} \overline{1}$ ] direction with distance of about $16.48 \mathrm{~nm}$ and; (III) volume of the dislocation clusters under the indenter tip changes a certain extent, as illustrated in Fig. 3(b) and (c). The region including the dislocation clusters under the indenter tip is usually called the plastic zone (PZ) of nanoindentation [30]. Dislocation evolution of modes I and II makes some contribution to the volume change of $\mathrm{PZ}$, but the volume change is also attributed to nucleation, growth, elimination and interactions of dislocations within PZ.

As a comparison, dislocation patterns before and after relaxation at the same loading step obtained with MD simulation is also demonstrated in Fig. 3(d) and (e) respectively. Before relaxation, there already exist some dislocations under the indenter tip and an emitted V-type dislocation located near the top surface. After 


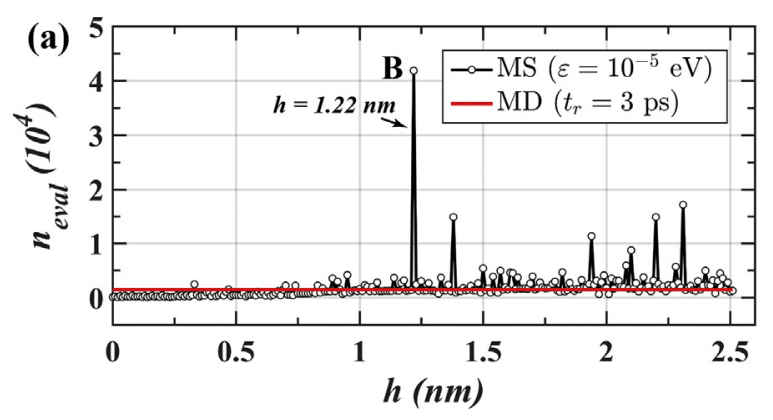

(b) MS (inital)

(c) MS (after relaxation)

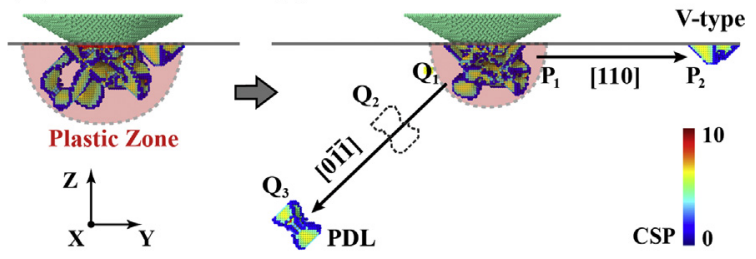

$\begin{array}{ll}\text { (d) } \mathrm{MD} \text { (inital) } & \text { (e) } \mathrm{MD} \text { (after relaxation) }\end{array}$

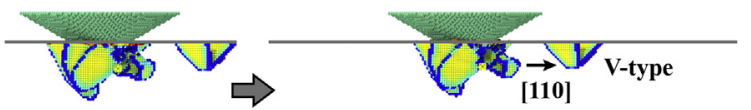

Fig. 3. (a) Times of force evaluation at each loading step in MS and MD simulations (b) and (c) show the dislocation patterns obtained with MS simulations before and after minimization at $h=1.22 \mathrm{~nm}$. (d) and (e) show the dislocation patterns obtained with MD before and after relaxation at $h=1.22 \mathrm{~nm}$.

relaxation, the V-type dislocation moves forward along [ $\left.\begin{array}{lll}1 & 1 & 0\end{array}\right]$ direction with a distance of about $1.02 \mathrm{~nm}$ which is much smaller than that of MS simulation. No recognizable change can be detected for the dislocation morphology inside PZ.

By tracking dislocation patterns in MS simulations, it is found that the computational cost of MS simulations is significantly affected by dislocation evolution. As the PDL in Fig. 3(c) propagating from Q1 to Q3, atoms near the path Q1 $\rightarrow$ Q3 change their positions step by step according to Eq. (2). For instance, when the PDL propagates to a position between Q1 and Q3 at iteration step $k$ of MS minimization, say Q2 as illustrated in Fig. 3(c), atoms around Q2 have changed their positions from previous perfect lattice to shape the PDL. At iteration step $k+1$, the PDL moves forward with a distance of $\Delta L$. The atoms around Q2 will go back to positions in perfect lattice, while atoms around the new location $(\mathrm{Q} 2+\Delta L)$ will change their positions accordingly to shape the PDL. The length of $\Delta L$ is determined by $\mathbf{d}_{k}$ and $\alpha_{k}$ in Eq. (2), while $\alpha_{k}$ is related to the current atomistic position vector $\mathbf{x}_{k}$ and force vector $\mathbf{f}_{k}$. Therefore, $\Delta L$ will be affected by the current atomistic configuration. Normally, the maximum atomistic displacement in an iteration step is about $2 \%$ of lattice constant $(\sim 0.007 \mathrm{~nm})$, so it takes tens of thousands iteration steps for the PDL moving from Q1 to Q3. The longer the moving distance of dislocation is, the more iteration steps and force evaluations are required. It is also the case for the V-type dislocation moving from P1 to P2.

The total iteration step is also dependent on the sequence of dislocation events. For example, if both the V-type dislocation and PDL propagated at the same time, $n_{\text {eval }}$ is determined by the longest propagation distance of the two dislocation events. However, if one happed after another, $n_{\text {eval }}$ is determined by the summation of the two distances. For the dislocation evolution of mode III, although there are a lot of dislocations within the PZ, the total distance of dislocation evolution is much shorter than that of mode I and II, so less iteration steps are required.

Based on the understanding of dislocation evolution-dependent $n_{\text {eval }}$ in MS simulations, the $n_{\text {eval }}-h$ relation as shown in Fig. 3(a) can be explained as follows. At elastic stage of indentation with $h<0.31 \mathrm{~nm}$, there is no dislocation; $n_{\text {eval }}$ is very small $(\langle 500)$. For most of loading steps when $h>0.31$, dislocation evolution is dominated by mode III with short evolution distance; so the $n_{\text {eval }}$ is less than 5000 in most cases. The $n_{\text {eval }}$ peaks similar to point B in Fig. 3(a) are corresponding to dislocation evolutions of modes I and/or II with long propagation distances.

\subsection{Fidelity of MD and MS simulations relevant to dislocation evolution}

Once the configuration and loading conditions are defined in nanoindentation, the relaxation time $\left(t_{r}\right)$ of MD and the resolution criterion $(\varepsilon)$ of MS are the key parameters that would affect the final simulation results. Definitely, the greater $t_{r}$ is, the slower loading velocity of MD simulation will be. The smaller $\varepsilon$ is, the more accurate MS simulation will be. Increasing $t_{r}$ or decreasing $\varepsilon$ will increase computational costs accordingly. In order to get reasonable results with less computational costs, it is important to understand how $t_{r}$ and $\varepsilon$ will affect simulation result of dislocation evolution.

Results from MS simulation with different $\varepsilon$ are plotted in Fig. 4. Basically, all $F$ increases with $h$ along with many mini-drops. In average the $F$ from simulation with larger $\varepsilon$ shows larger than that from simulation with smaller $\varepsilon$.

Moreover, dislocation distributions at the last loading step obtained from simulation with $\varepsilon=10^{-3}$ and $10^{-5} \mathrm{eV}$ are significantly different, as shown in Fig. 4(b) A and B. There are more dislocation clusters located around the PZ for $\varepsilon=10^{-3} \mathrm{eV}$, while there are more PDLs distributed in the substrate for $\varepsilon=10^{-5} \mathrm{eV}$. The different dislocation distribution also comes along different computational costs. Specifically, for the three MS simulations with $\varepsilon=10^{-3}, 10^{-4}$ and $10^{-5} \mathrm{eV}$, ratio of $N_{\text {eval }}$ at the final loading step $(h=2.53 \mathrm{~nm})$ is about $1: 10: 30$. Since the dislocation distribution is history-dependent, it is hard to tell when and how the difference of dislocation distribution comes from. In order to understand the origin of the difference, additional MS simulations with different $\varepsilon$

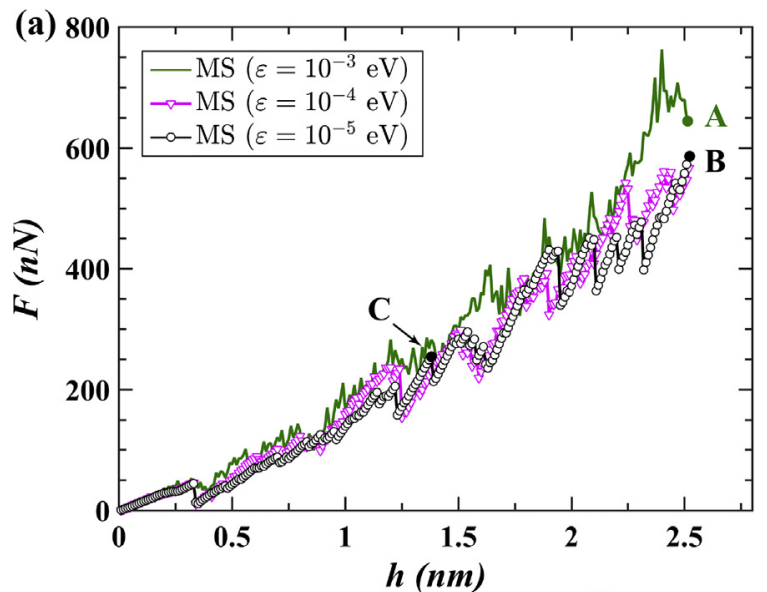

(b)

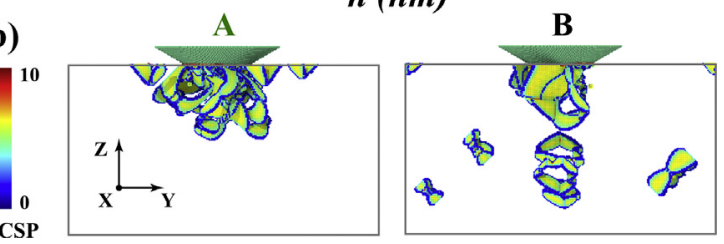

Fig. 4. (a) $F-h$ curves obtained from MS simulations with different $\varepsilon$ settings. (b) Dislocation distribution at the last loading step $(h=2.53 \mathrm{~nm})$ from MS simulations with $\varepsilon=10^{-3}$ and $1 \times 10^{-5} \mathrm{eV}$ respectively. 
starting from the same initial atomistic configuration (point $C$ in Fig. 4(a)) are performed. The results are demonstrated in Fig. 5.

At the initial state A in Fig. 5(b), there is a ready-to-emit PDL located at $\mathrm{Q}_{1}$. At the next loading step, the indenter tip moves from $h=1.37 \mathrm{~nm}$ to $1.38 \mathrm{~nm}$. When the system is minimized with $\varepsilon=10^{-3} \mathrm{eV}$, no significant changes is detected from the dislocation distribution B in Fig. 5(b), but the $F$ increases from 255.3 to 283.7 $\mathrm{nN}$ accordingly. In the dislocation distribution $\mathrm{C}$ obtained in simulation with $\varepsilon=10^{-4} \mathrm{eV}$, the PDL has been emitted and moved from $\mathrm{Q}_{1}$ to $\mathrm{Q}_{2}$. However, the $F$ decreases from 255.3 to $216.9 \mathrm{nN}$. For the dislocation distribution D obtained with $\varepsilon=10^{-5} \mathrm{eV}$, the PDL has been emitted and moved from $Q_{1}$ to $Q_{3}$ with distance longer than that in C. The corresponding $F$ decreases from 255.3 to $213.6 \mathrm{nN}$ which is slightly smaller than that of $216.9 \mathrm{nN}$ at C. There is also a noticeable difference of dislocation at location $S_{1}$ in $C$ and $D$. The results indicate that when MS simulation is performed with smaller $\varepsilon$, it is "easier" for dislocations to be emitted from PZ and move further. In other words, dislocations can be developed more maturely in simulations with higher accuracy.

The connection between the variation of $F$ and dislocation states can be understood based on two points. Firstly, the value of $F$ is mainly determined by the energy states or dislocation states within PZ. Difference of dislocation conditions far away from PZ has small effect on F. Actually, based on the similar understanding, Nix and Gao have developed a theory to estimate hardness of material from dislocation states in PZ [31]. Secondly, energy change in PZ is due to the competition between the effects of indentation and dislocation evolution. Specifically, indentation of the tip will increase energies in $\mathrm{PZ}$ and therefore lead to the increase of $F$, while emission of dislocations from PZ will release energies in $\mathrm{PZ}$ and decrease $F$.

From A to B in Fig. 5(b), no dislocation is emitted from PZ and dislocations within PZ is not maturely developed due to low accuracy, so $F$ increases due to the effect of indentation. However, from A to $C$, there is a PDL emitted from $Q_{1}$ to $Q_{2}$. The decrease of $F$ indicates that the energy released in PZ due to emission of PDL is much

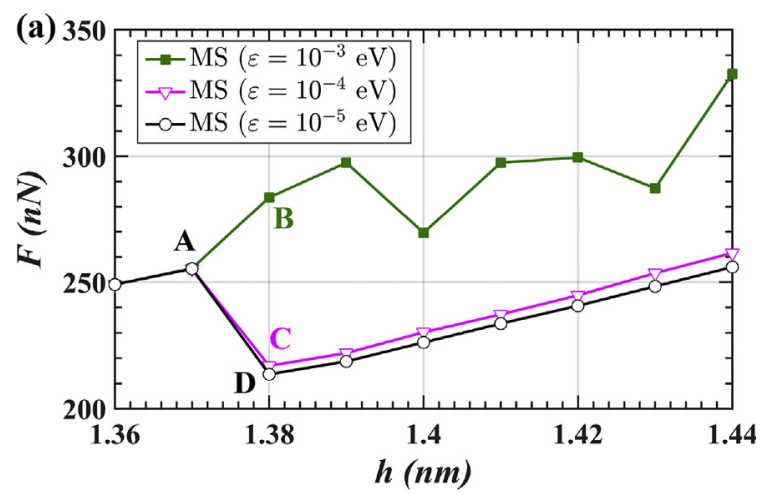

(b)

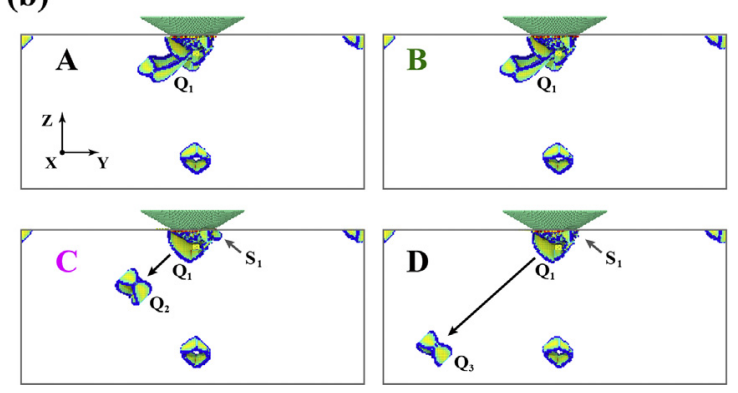

Fig. 5. (a) $F$ - $h$ curves obtained from MS simulations with different $\varepsilon$ starting from the same initial atomistic configuration. (b) Dislocation distribution at point A, B, C, and $D$ in (a) respectively. larger than the energy collected due to indentation with $\Delta h=0.01 \mathrm{~nm}$. It is also the similar condition for the case from A to $\mathrm{D}$. Although the final position of the PDL in C and D is quite different, the value of $F$ at $C$ and D in Fig. 5(a) is very close. It is because once the PDL has moved far away from $\mathrm{PZ}$, the indenter tip cannot "feel" its further evolution. Therefore, further propagation of the PDL from $Q_{2}$ to $Q_{3}$ does not affect the variation of $F$ too much. The small separation between the value of $F$ at $C$ and D in Fig. 5(a) is because dislocations within PZ at D are developed more maturely than that at $C$ due to smaller $\varepsilon$ setting which can be seen from the dislocation difference at $S_{1}$ in $C$ and $D$.

Results in Fig. 4 can be explained based on the same understandings of Fig. 5 accordingly. Firstly, in average all the $F$ increases with $h$, because the total energy in PZ increases due to successive indentation of the tip. Emission of dislocations from PZ will leads to the mini-drops of $F$, but it does not happen in every loading step. Secondly, when MS simulation is performed with smaller $\varepsilon$, there are more chances for dislocations to emit from PZ which leads to smaller $F$ observed in Fig. 4(a). Thirdly, the accumulated $\varepsilon$ dependent dislocations at each loading step leads to the final different dislocation distributions in Fig. 4(b). Therefore, more computational cost is required in MS simulation with higher resolution.

Similar phenomenon can be observed in MD simulations with different $t_{r}$ as shown in Fig. 6. At $h=2.53 \mathrm{~nm}$, the $F$ from MD simulation with $t_{r}=0.2 \mathrm{ps}$ is $941.0 \mathrm{nN}$ which is much larger than 517.8 $\mathrm{nN}$ of $t_{r}=10 \mathrm{ps}$. It is because dislocations in the substrate is not maturely developed with smaller $t_{r}$, as shown in Fig. 6(b). Since the computational cost of MD simulation is totally determined by $t_{r}$, for the three MD simulations with $t_{r}=0.2,3$ and $10 \mathrm{ps,}$ $N_{\text {eval }}$ ratio at the final loading step is $0.2: 3: 10=1: 15: 50$.

Although the dislocation distribution, especially the distribution of PDLs in the substrate, in MS simulation with $\varepsilon=10^{-5} \mathrm{eV}$ (B in Fig. 4(c)) and MD simulation with $t_{r}=10$ ps (B in Fig. 6(b)) is quite different, the $F$ - $h$ curves of them (Fig. 2(a)) are consistent with each other. It indicates that dislocations in the $\mathrm{PZ}$ of both MD and MS simulations are developed to the similar extent, no matter how different the dislocation patterns are and how many PDLs have been emitted. It should be mentioned that dislocation
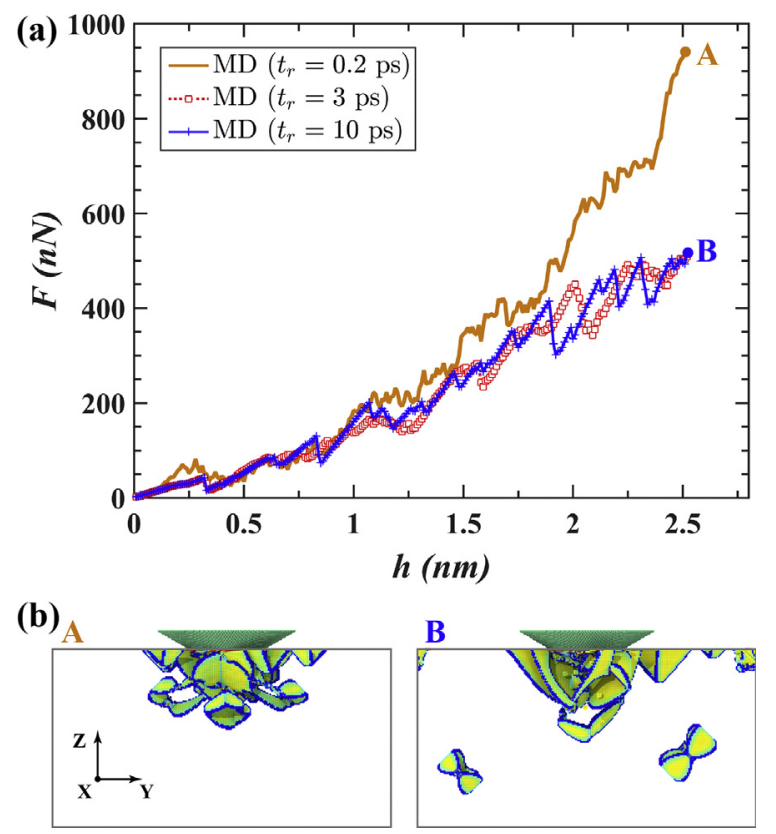

Fig. 6. (a) $F$ - $h$ curves obtained from MD simulations with different $t_{r}$ settings. (b) Dislocation distribution at the last loading step from simulations with $t_{r}=0.2$ and 10 ps respectively. 
evolution is history-dependent and stochastic during simulations, so there will be small dispersion between the $F$ - $h$ curves of MD and MS.

\subsection{Choice between MD and EM-based methods in simulation relevant to dislocation evolution}

Based on the above insights, the following points should be considered when making choice between MD and EM-based methods in simulations relevant to dislocation evolutions:

(1) In the current simulation of nanoindentation system, MS with $\varepsilon=10^{-5} \mathrm{eV}$ can yield good results with maturely developed dislocations; and the computational cost is acceptable in comparison with that of MD simulations. However, computational cost of MS is strongly dependent on dislocation events and atomistic configurations. It is hard to derive an explicit and general expression of computational cost including effects of dislocation states and convergence resolution $(\varepsilon)$. When dislocation events are not predominant in comparison with elastic deformation in a given quasi-static problem, MS simulation with adequate accuracy could provide good results with competitive computational efficiency. Nevertheless, if dislocation evolution is intense in a problem, computational cost of MS will grow with dislocation events and is unpredictable. EM-based multi-scale methods will encounter the same problem when deal with large scale dislocation evolution. Therefore, designing new minimization algorithms that enable to get rid of inessential microscopic details is a fundamental task in order to further improve computational efficiency of multi-scale methods.

(2) Unlike MS with intrinsic support for quasi-static simulation of dislocation evolution, in MD simulations a "quasi-static" process is effected by increasing the relaxation time $\left(t_{r}\right)$. Although the increase of $t_{r}$ will dramatically increase computational cost, MD with an appropriate $t_{r}$, e.g. 3 ps in the current simulation of nanoindentation, could provide similar results to that of MS simulations. A benefit of using MD to simulate dislocation related problems is that the computational cost is predictable and controllable. Moreover, MD can provide a real dynamic simulation of dislocation evolution at finite temperature. However, a pre-specified relaxation time in MD cannot guarantee that dislocations are maturely developed at every loading step. For a quasistatic problem, trial MD simulations can be performed along with MS simulation in order to choose a good $t_{r}$ with acceptable efficiency and fidelity.

(3) MD with larger $t_{r}$ and MS with smaller $\varepsilon$ will give more opportunity for dislocations to evolve at a specific loading condition, yet sometimes maturely developed dislocation is not necessary. Take the simulation of nanoindentation for example, an appropriate $t_{r}$ or $\varepsilon$ can be used to save computational cost as long as the $F-h$ response meets requirements, since the $F-h$ relation is mainly dependent on dislocation conditions in PZ. However, if we focus on microscopic details of dislocation evolution and distribution in the whole system, simulations with high resolution settings should be used.

\section{Summary}

Simulations of nanoindentation with dislocation evolutions are performed by means of MD with different relaxation time $\left(t_{r}\right)$ and EM-based method (MS) with different convergence resolution ( $\varepsilon$ ) to investigate the computational efficiency and fidelity of the two methods. Although the relations of force vs. indentation depth
(F-h) obtained in MD with $t_{r}=3$ ps and MS with $\varepsilon=10^{-5} \mathrm{eV}$ are consistent with each other, the computational cost is significantly different. Because $t_{r}$ is pre-specified, the computational cost of MD increases linearly with indentation depth and has nothing to do with how complicated the dislocation evolutions are. On the contrary, computational cost of MS is strongly dependent on dislocation events and increases nonlinearly with indentation depth. Further investigation reveals that the computational cost paid in MS with minimization algorithm is proportional to the distance where dislocation reaches. Particularly, the evolution of PDLs or V-type dislocations with long propagation distance in substrate will dramatically lead to high computational consumption.

As for the dislocation distributions, some results obtained with MS and MD are similar but some are different. It is found that there will be more PDLs and V-type dislocations emitted from plastic zone within the substrate when MD simulations is performed with longer relaxation time $t_{r}$ and MS simulations with higher resolution, i.e. smaller $\varepsilon$, but the MS usually presents even more and far-reaching dislocations. Although the emitting and propagating of PDLs and V-type dislocations will lead to the mini-drops on $F$ - $h$ curve, the increasing trend of $F$ with indentation depth is mainly governed by the dislocation conditions in the plastic zone of the substrate underneath the indenter only. So the simulations of MD with $t_{r}=3 \mathrm{ps}$ and MS with $\varepsilon=10^{-5} \mathrm{eV}$ yield similar $F-h$ curves but with different dislocation distributions. Based on this insight, strategies regarding the choice between MS and MD in simulations relevant to dislocation evolutions are discussed respectively.

Actually, we meet a practical challenge now. That is, in order to further improve both computational efficiency and fidelity in molecular simulation of dislocation evolutions, a new MD algorithm with adaptive relaxation time, or some smart minimization algorithms of EM-based multi-scale methods enable to get rid of inessential microscopic details must be developed.

\section{Acknowledgements}

This work was supported by the National Natural Science Foundation of China [grant number 11672298, 11432014 and 11372316], the Strategic Priority Research Program (B) of the Chinese Academy of Sciences [grant number XDB22040501]. Computations are performed on the ScGrid of Supercomputing Center, Computer Network Information Center of Chinese Academy of Sciences and LNMGrid of the State Key Laboratory of Nonlinear Mechanics.

\section{References}

[1] V. Yamakov, D. Wolf, S.R. Phillpot, A.K. Mukherjee, H. Gleiter, Nat. Mater. 1 (2002) 45.

[2] C. Domain, G. Monnet, Phys. Rev. Lett. 95 (2005) 215506.

[3] S. Groh, E.B. Marin, M.F. Horstemeyer, D.J. Bammann, Model. Simul. Mater. Sci. Eng. 17 (2009) 75009.

[4] T.A. Parthasarathy, S.I. Rao, D.M. Dimiduk, Philos. Mag. A 67 (1993) 643.

[5] A. Gouldstone, N. Chollacoop, M. Dao, J. Li, A. Minor, Y. Shen, Acta Mater. 55 (2007) 4015.

[6] C.J. Ruestes, E.M. Bringa, R.E. Rudd, B.A. Remington, T.P. Remington, M.A. Meyers, Sci. Rep. 5 (2015) 16892

[7] P. Xiao, J. Wang, R. Yang, F. Ke, Y. Bai, Comput. Mater. Sci. 98 (2015) 70.

[8] D. Bhattacharya, J. Cheng, Prot. Struct. Funct. Bioinform. 81 (2013) 119.

[9] J.W. Kang, H.J. Hwang, Nanotechnology 12 (2001) 295.

[10] H. Wang, M. Hu, M. Xia, F. Ke, Y. Bai, Int. J. Solids Struct. 45 (2008) 3918.

[11] J. Wang, P. Xiao, M. Zhou, Z.R. Wang, F.J. Ke, J. Appl. Phys. 107 (2010) 23512.

[12] P. Xiao, J. Wang, F.J. Ke, Y.L. Bai, Compos. Part B Eng. 43 (2012) 57.

[13] E.B. Tadmor, M. Ortiz, R. Phillips, Philos. Mag. A 73 (1996) 1529.

[14] R.E. Rudd, J.Q. Broughton, Phys. Stat. Solidi 217 (2000) 251.

[15] S.P. Xiao, T. Belytschko, Comput. Methods Appl. Mech. Eng. 193 (2004) 1645.

[16] D. Qian, G.J. Wagner, W.K. Liu, Comput. Methods Appl. Mech. Eng. 193 (2004) 1603.

[17] D.K. Datta, R.C. Picu, M.S. Shephard, Int. J. Multiscale Comput. Eng. 2 (2004) 401. 
[18] Q. Yang, E. Biyikli, A.C. To, Comput. Methods Appl. Mech. Eng. 258 (2013) 26.

[19] R.E. Miller, E.B. Tadmor, Model. Simul. Mater. Sci. Eng. 17 (2009) 53001.

[20] J. Chen, P. Ming, J.Z. Yang, Commun. Comput. Phys. 15 (2014) 470.

[21] J. Chen, P. Ming, Commun. Comput. Phys. 10 (2011) 70.

[22] L.E. Shilkrot, R.E. Miller, W.A. Curtin, J. Mech. Phys. Solids 52 (2004) 755.

[23] E. Biyikli, A.C. To, J. Comput. Phys. 328 (2017) 27.

[24] Y. Mishin, D. Farkas, M.J. Mehl, D.A. Papaconstantopoulos, Phys. Rev. B 59 (1999) 3393.
[25] Y. Mishin, M.J. Mehl, D.A. Papaconstantopoulos, A.F. Voter, J.D. Kress, Phys. Rev. B 63 (2001) 224106

[26] M.C. Payne, M.P. Teter, D.C. Allan, T.A. Arias, J.D. Joannopoulos, Rev. Mod. Phys. 64 (1992) 1045

[27] S. Plimpton, J. Comput. Phys. 117 (1995)1.

[28] A. Hasnaoui, P.M. Derlet, H. Van Swygenhoven, Acta Mater. 52 (2004) 2251.

[29] C.L. Kelchner, S.J. Plimpton, J.C. Hamilton, Phys. Rev. B 58 (1998) 11085.

[30] K. Durst, B. Backes, M. Göken, Scr. Mater. 52 (2005) 1093.

[31] W.D. Nix, H. Gao, J. Mech. Phys. Solids 46 (1998) 411. 